\title{
Traducción jurídica, mujeres indígenas y acceso a una vida libre de violencia
}

Legal translation, indigenous women and the access to a life free of violence

ISSN 2071-8748

E-ISSN 2218-3345

(c) (1) \$(

BY NC SA

DOI: https://doi.org/10.5377/entorno.v0i69.9567

URI: http://hdl.handle.net/11298/1161

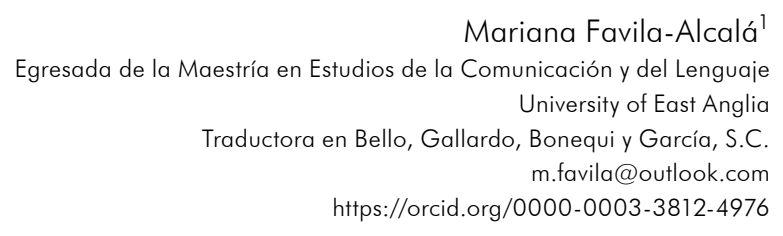

Recibido: 6 de enero 2020 Aceptado: 27 de junio de 202

\section{Resumen}

En 2017 se publicó la traducción de la Ley de Acceso de las Mujeres a una Vida Libre de Violencia del Estado de México a cinco lenguas indígenas prevalecientes en dicha entidad. Si bien esta acción pudiera percibirse como progresista e incluyente, si se considera que México se ha caracterizado por tensiones lingüísticas, jurídicas e identitarias, resulta imperativo reflexionar, de manera crítica, en torno al papel que juega y jugará la traducción en el acceso de las mujeres indígenas a una vida libre de violencia. Para ello, en la primera etapa de esta investigación se consideró la concepción sociojurídica de los pueblos originarios, el lugar que ocupan las lenguas nacionales y la situación particular que enfrentan las mujeres indígenas. Además, se entrevistó a tres personas clave en la realización de la traducción de la ley en cuestión y, por último, se analizó la función de la traducción con base en el contexto en el que

\section{Abstract}

In 2017, the translation of the Ley de Acceso de las Mujeres a una Vida Libre de Violencia del Estado de México (Law of Access of Women to a Life Free of Violence of the State of Mexico) was published into five of the most prevailing indigenous tongues in said [judicial] identity. Although this action could be perceived as progressive and inclusive, if one takes into consideration that Mexico has been characterized by identity, judicial and linguistic tensions, it is imperative to critically reflect around the role that translation plays, and will play, in relation to the access to a life free of violence for the indigenous women. With this purpose, the first stage of this investigation took into consideration the socio-legal conception of the originary peoples, the place that the national tongues take and the particular situation indigenous women face. Also, three key people were interviewed for the translation of this

1 Maestría en Estudios de la Comunicación y del Lenguaje; ORCID: Mariana Favila Alcalá (https://orcid.org/0000-0003-3812-4976) 
esta se inserta. La información recabada sugiere que el papel de la traducción es ambivalente. Por un lado, puede servir como instrumento de empoderamiento; $y$, por otro, es una herramienta de perpetuación del etnocentrismo jurídico y de la violencia racista que aqueja tanto a las instancias gubernamentales como a la sociedad civil. En la segunda etapa, que sigue en desarrollo, se pretende entrevistar a un grupo representativo de mujeres mazahuas para conocer el grado de socialización de los términos propuestos por el traductor, así como indagar en el impacto que la traducción ha tenido en sus vidas.

\section{Palabras clave}

Mujeres - México - Derechos humanos; Delitos contra la mujer - México; Indígenas - Situación legal - México; Traducción jurídica - México; Derecho lingüístico - México. law; and last, an analysis was made of the function of said translation based on the context where it is inserted. The collected data suggests that the role of this translation is ambivalent. This can be seen from two different perspectives: number one, as a tool for empowerment; number two, as a tool for the perpetuation of a judicial ethnocentrism and violent racism that troubles both the government entities and the civil society. In the second stage, still under development, a representative group of Mazahua women will be interviewed in order to know the degree of socialization of the terms proposed by the translator; in addition, the impact of the translation on their lives will be inquired.

\section{Keywords}

Women - Mexico -Human rights; Crimes against women - Mexico; Indigenous peoples - Legal situation - Mexico; Linguistic rights - Mexico.

\section{Introducción}

Por lo general, se piensa que el disfrute de derechos está al alcance de todas las personas, sin ningún tipo de distinción; sin embargo, la realidad dista mucho de ser así: a un gran número de comunidades y de personas se les ha negado y se les sigue negando el ejercicio de sus derechos por su color de piel, género y/o lengua (Chenaut, 2007; Conley y O'Barr, 2005; Sierra, 2004). Prueba de ello es que, si bien México ratificó el Convenio $169^{2}$ hace casi tres décadas, las medidas jurídicas que el estado mexicano ha tomado para darle cumplimiento poco han hecho para erradicar la subordinación y discriminación que han padecido los pueblos y comunidades indígenas desde la llegada de los conquistadores españoles (Hernández y Ortiz, 2003; López Bárcenas, 2010; Sierra, 2005).

Una de esas medidas es, por ejemplo, la promulgación de la Ley General de Derechos Lingüísticos de los Pueblos Indígenas en el año 2003. El propósito de esta ley es, por un lado, lograr que se reconozcan, estudien, protejan y promuevan las lenguas indígenas; y, por otro, que el contenido de cualquier ley, norma, programa o servicio que afecte la vida de los pueblos y comunidades indígenas se haga de su conocimiento en la lengua de la que sean hablantes. Sin embargo, Vázquez (2009) y Giovine (2010) hacen ver que la ley en ningún momento especifica qué acciones se llevarán a cabo para lograr sus objetivos ni precisa quiénes se encargarán de emprender tales acciones ni con qué recursos lo harán; por tanto, para Vázquez (2009) y Giovine (2010), la aplicación de esta ley ha sido precaria e insuficiente.

2 El Convenio 169 fue elaborado por la Organización Internacional del Trabajo (OIT) y constituye el instrumento jurídico más importante a escala internacional en materia de derechos de pueblos y comunidades indígenas, puesto que obliga a los estados miembro de la olt a respetar y garantizar el ejercicio de los derechos colectivos de pueblos y comunidades indígenas (López Bárcenas, 2006), que incluyen la preservación de sus lenguas y el reconocimiento de sus formas de organización social, de la validez de sus sistemas jurídicos y de su autonomía política (De la Peña, 2006). Para ahondar en el tema del derecho a la autodeterminación de los pueblos indígenas, ver Correas (2012) y Villoro (2017). 
Además, si bien esta ley establece que el español y las lenguas indígenas son 'lenguas nacionales' $y$, por ende, tienen el mismo rango, el español sigue siendo la lengua imperante en todos los ámbitos de la vida pública, pues se le relaciona con el progreso, la modernidad, el prestigio y la riqueza. En consecuencia, en vista de que las lenguas indígenas se piensan como sinónimo de inferioridad, ignorancia, pobreza y falta de desarrollo, la discriminación es la principal causa por la cual una buena parte de los pueblos y comunidades indígenas han optado por dejar de hablar su lengua, o bien la utilizan solo en la esfera privada (Canuto, 2013; Giovine, 2010).

De cualquier manera, en apego a la Ley General de Derechos Lingüísticos de los Pueblos Indígenas, en el año 2017 una dependencia gubernamental elaboró y publicó la traducción de la Ley de Acceso de las Mujeres a una Vida Libre de Violencia del Estado de México a las lenguas indígenas que prevalecen en dicha entidad: matlatzinca, mazahua, náhuatl, otomí y tlahuica. El propósito era informar a las mujeres indígenas del contenido de la ley y, de esa forma, garantizar que estén en posibilidades de identificar la violencia y de activar los mecanismos que las protegen de ella. Esta acción se antoja progresiva e incluyente; sin embargo, resulta imperativo reflexionar, de manera crítica, en torno al papel que juega y jugará la traducción de una ley en materia de violencia de género en la vida de las mujeres indígenas si se considera que (a) el derecho está impregnado de la historia, tradiciones y aspiraciones de la sociedad de la que emana (Gémar, 2005; Gibbons, 2003; Martí, 2004; Potapouchkine y Haenisch, 2003; Wolff, 2011); (b) el discurso jurídico es complejo a nivel sintáctico y de léxico, razón por la cual en ocasiones se considera intraducible (Conley y O'Barr, 2005; Mooney, 2014; Tiersma, 1999); y (c) la totalidad de las lenguas indígenas en México se encuentra en peligro de desaparición (Instituto Nacional de Lenguas Indígenas, 2010).

En relación con lo anterior, cabe decir que un número importante de documentos jurídicos se ha traducido a las lenguas indígenas que coexisten con el español en México; sin embargo, este fenómeno ha recibido poca atención tanto de la academia como de las autoridades gubernamentales (Meneses, 2004). Por tal motivo, este proyecto busca también aunar a la investigación relativa a las lenguas minorizadas $y$, por medio de los estudios descriptivos de traducción, ${ }^{3}$ pretende contribuir a la descolonización y reconceptualización de la práctica traductora en Nuestramérica.

\section{Metodología}

Producto del encuentro entre mundos dispares -el indígena y el europeo-, con base en la recién nacida idea de superioridad racial de quienes provenían de Europa, las poblaciones originarias que habitaban en lo que hoy se denomina América fueron objeto de estratificación, marginación y subordinación (Gándara Carballido, 2019). Así, desde la llegada de los conquistadores españoles, el término indígena se ha utilizado no solo como una etiqueta que pretende equiparar y homogeneizar las prácticas culturales, lingüísticas y jurídicas que coexisten en el territorio que hoy Ilamamos México (Aguilar Gil, 2018), sino como sinónimo de ignorancia y atraso (Oehmichen, 2005).

Con ello en mente, en vista de que el proceso traductor se ve influenciado por factores contextuales (Wolf, 2007), esta investigación consideró las características socioculturales y político-ideológicas del contexto que rodea la traducción del español al mazahua ${ }^{4}$ de la Ley de Acceso de las Mujeres a una Vida Libre de Violencia del Estado de México. Para ello, se hizo una revisión crítica de literatura en materia antropológica (Aguilar Gil, 2018 y 2019; Hernández y Ortiz, 2003; Oehmichen, 2005; Villoro, 2017), jurídica (Correas, 2012; Fernández Chagoya, 2016; Gándara Carballido, 2019; López Bárcenas, 2010; Sierra, 2005), lingüística (Aguilar Gil, 2018; Vázquez, 2009) y de género (Alberti, 2004; Cerna Villagra, 2018; Espinosa Miñoso, Gómez Correal y Ochoa Muñoz, 2014; Ochoa Muñoz, 2019; Sierra, 2004 y 2009).

Aunado a ello, se identificaron y documentaron los obstáculos que surgieron durante la realización de la

3 Los estudios descriptivos de traducción pretenden reseñar qué puede ser y qué es la traducción en circunstancias específicas; analizan la práctica traductora con base en el producto, el proceso, las personas participantes y la función que dicha práctica cumple (Toury, 1991).

4 Por razones de espacio y tiempo, esta investigación involucró nada más al traductor de la lengua mazahua, una de las cinco lenguas a las que fue traducida la ley en cuestión. 
traducción, así como las estrategias que se implementaron para superar tales obstáculos. Para lograrlo, con base en el modelo propuesto por Nord (1988), ${ }^{5}$ se entrevistó, de manera remota, ${ }^{6}$ a tres personas que jugaron un papel clave en la realización de la traducción: el vocal ejecutivo de la dependencia gubernamental que gestionó la traducción (Monroy, 2017); la directora adjunta del Área de Desarrollo Cultural Indígena de dicha dependencia (Cano, 2017); y el traductor, un antropólogo mazahua certificado por el Instituto Nacional de Lenguas Indígenas, para desempeñarse como intérprete y traductor (Segundo, 2017). Por último, con base en la información recabada, se analizó el papel que parece jugar la traducción en el acceso de las mujeres indígenas a una vida libre de violencia.

\section{Resultados y aportes}

Condición sociojurídica de pueblos y comunidades indígenas

El multiculturalismo en México constituye una realidad compleja que ha sido objeto de negación constante (Aragón, 2007). En ese sentido, la antropología jurídica sugiere que, si bien la reforma constitucional de 2001 reconoce la diversidad cultural en México y condena toda forma de discriminación, la manera en que dicha reforma fue concebida y redactada perpetúa la subordinación indígena, pues no supone ningún cambio estructural para el ejercicio del derecho a la libre autodeterminación de los pueblos y comunidades originarios (De la Peña, 2006; Hernández y Ortiz, 2003; López Bárcenas, 2010; Sierra 2005). Aunado a ello, si bien otorga reconocimiento a los sistemas jurídicos indígenas, estos deben sujetarse a la discreción de las autoridades estatales (Adonon, 2009; Aragón, 2007; González, 2010; Sierra, 2004).

Puede concluirse entonces que la sociedad mexicana y su gobierno insisten en favorecer prácticas jurídicas occidentales so pretexto de que los sistemas jurídicos indígenas son primitivos y subdesarrollados (Aragón, 2007; Correas, 2012; Sierra, 2005). En consecuencia, los pueblos y comunidades indígenas deben seguir luchando contra el racismo, etnocentrismo y monismo jurídico que constituyen las piedras fundantes del estado mexicano, que, todavía hoy, se piensa homogéneo (Hernández y Ortiz, 2003).

\section{Mujeres indígenas}

México se erigió como país soberano e independiente con base en el 'mito del mestizaje', el cual impuso la superioridad de personas y prácticas —lingüísticas, políticas y jurídicasoccidentalizadas (De la Peña, 2006; Hernández y Ortiz, 2003). Al ser quienes daban a luz a las personas mestizas, se esperaba que las mujeres indígenas no dejaran de ser quienes eran, es decir, mujeres tradicionales, naturales, no modernas y de piel morena. Así, fueron condenadas a la marginación que enfrentan todavía hoy por la carga negativa que se ha asignado no solo a la categoría 'mujer', sino a la categoría indígena como sinónimo de atraso y pobreza (CIDH, 2017; Gall, 2004; Segato, 2014).

La sociedad mazahua, como muchas otras comunidades indígenas, pudiera catalogarse como patriarcal, puesto que las mujeres no tienen permitido poseer tierras; se les excluye de los procesos de toma de decisiones; se espera que obedezcan a sus esposos y que se dediquen, primordialmente y con diligencia, a las tareas del hogar. Además, sufren violencia física y verbal, pero rara vez abandonan a sus parejas no solo porque consideran que la violencia es algo 'normal' o privado, sino porque separarse de la pareja es mal visto en la comunidad y la mayoría depende económicamente de su marido (Oehmichen, 2005).

Aunado a lo anterior, Olivares Ferreto et al. (2014) identificaron una amplia gama de violencias que sufren las mujeres mazahuas que deciden asistir a la universidad: (1) reciben comentarios discriminatorios - por parte del estudiantado, profesorado y equipo administrativo- por el hecho de ser indígenas; (2) se les desvaloriza y estereotipa por el hecho de ser mujeres (el profesorado les asigna tareas asociadas con la feminidad y les prohíbe tareas

5 El modelo de Nord (1988), que forma parte de la corriente sociológica de la traducción, es un modelo comunicativo que propone que el estudio y análisis de la traducción debe considerar factores internos y externos (es decir, elementos lingüísticos y contextuales), y sugiere que los métodos y técnicas de traducción dependerán, en gran medida, de la función del texto meta (es decir, el fin para el cual se realiza la traducción).

6 Las entrevistas se llevaron a cabo vía remota debido a que, según Saldahna y o’Brien (2013), la interacción asíncrona permite que las respuestas sean mucho más amplias y ricas en detalle, puesto que quienes las responden pueden hacerlo cuando les resulte más conveniente y pueden, asimismo, corroborar datos previos a ello. Así, la validez de la información recabada es mucho mayor. 
asociadas con la masculinidad, mientras que la familia y la comunidad esperan que abandonen los estudios para que cumplan con el papel que tradicionalmente les corresponde como mujeres); (3) reciben menos apoyo económico de sus familias en comparación con los varones; y (4) han sido atacadas sexualmente por compañeros, profesores y autoridades educativas. En consecuencia, muchas jóvenes optan por abandonar los estudios, con lo cual se mantienen en la exclusión y marginación educativa y política (Cerna Villagra, 2018).

De manera similar, en su relación con las instituciones $\mathrm{y}$ autoridades, las mujeres indígenas se enfrentan con ideologías genéricas que perpetúan su subordinación y dan privilegio a sus victimarios, pues se minimiza la violencia que sufren; reciben sermones sobre el papel que deben jugar mujeres y hombres en tanto que tales o se les sugiere aguantar la violencia que reciben de sus maridos (Alberti, 2004; Oehmichen, 2005; Sierra, 2004); asimismo, se les impide elegir la justicia —indígena o estatal ${ }^{7}$ que ellas consideran mejor para la resolución de los conflictos en que se ven involucradas y no siempre cuentan con un/a intérprete -incluso se les obliga a declarar en español(EQUIS et al., 2019; CIDH, 2017).

Además, según la Comisión Interamericana de Derechos Humanos (CIDH, 2017), a pesar de contar con leyes y protocolos de atención en casos de violencia de género, el estado no ha logrado garantizar los derechos de las mujeres indígenas, puesto que no cuenta con "sistemas o procedimientos para recabar pruebas que [incorporen] la visión, cultura y perspectiva de las mujeres indígenas, [de manera que se ve imposibilitado para abordar] las necesidades particulares [de estas mujeres]" (p. 106). Al respecto, EQUIS et al. (2019) señalan que "es práctica común para la mayoría de las encuestas sobre violencia y acceso a la justicia que, si la mujer entrevistada no habla español, la entrevista se concluye sin que se capture su testimonio" (p. 15).

Aunado a lo anterior, a pesar de ser también victimario en vista de las omisiones mencionadas, ${ }^{8}$ el estado criminaliza a las comunidades, pueblos y autoridades indígenas por considerar que sus prácticas son inherentemente violatorias de los derechos humanos de las mujeres ${ }^{9} y$, por ello, les niega capacitación en la materia, a pesar de que las propias mujeres indígenas han expresado su deseo de que el derecho indígena cuente con mecanismos que le permitan brindar soluciones en casos de violencia de género en sus comunidades (EQUIS et al., 2019).

En ese sentido, cabe señalar que existen colectivos de mujeres indígenas que no solo están denunciando e iniciando procedimientos judiciales debido a la violencia que sufren como mujeres (Chenaut, 2007), sino que se han apropiado también del discurso que cuestiona los papeles de género (Sierra, 2009). No obstante, Araiza (2006) y Chenaut (2007) observaron que las mujeres indígenas que participan activamente en la defensa de sus derechos tienden a sufrir niveles más altos de violencia física y verbal, pues se les considera transgresoras de los papeles de género y se piensa que ponen en riesgo la totalidad del movimiento indígena (Chenaut, 2007). Por ello, el estado mexicano se siente con el poder y la facultad de intervenir y de anular el derecho indígena (Aragón, 2007). Al respecto, cabe decir que la lucha política de las mujeres indígenas - feministas o no- $-{ }^{10}$ tiene dos componentes: por un lado, exigen que se respete el derecho que tanto ellas como sus pueblos tienen en cuanto al diseño de su idea de 'ser pueblo' (Villoro, 2017); $y$, por otro, buscan que las tradiciones de sus comunidades evolucionen de manera tal que sus derechos económicos, políticos y sociales no se vean mermados (Cerna Villagra, 2018; Espinosa et al., 2014).

7 Cabe decir que, según EQUIS et al. (2019), un importante número de mujeres indígenas desconoce la existencia de las autoridades estatales, o bien estas se encuentran geográficamente lejos del hogar de las mujeres; no se acercan a ellas para pedir justicia por desconocimiento o por no contar con los recursos para ello.

8 La Suprema Corte de Justicia de la Nación emitió dos protocolos: uno para la impartición de justicia en casos que involucren personas, comunidades y pueblos indígenas; y otro para juzgar con perspectiva de género. Sin embargo, ninguno de ellos es de carácter obligatorio para los órganos jurisdiccionales (CIDH, 2017).

9 A manera de ejemplo, cabe mencionar que el artículo 420 del Código Nacional de Procedimientos Penales prohíbe expresamente que la justicia comunitaria conozca de casos de violencia contra las mujeres.

10 Muchas mujeres indígenas no se identifican como feministas, puesto que consideran que se trata de un movimiento burgués alejado de su realidad y de sus necesidades (Espinosa et al, 2014). 


\section{Reconceptualización de la traducción}

Durante mucho tiempo, la traducción se concibió como una actividad inmune a todo lo que ocurría a su alrededor (Bassnett, 2014; Davies, 2011; Genztler, 2002; Pym, 2006; Yameng 2007). Sin embargo, con el giro cultural de la década de 1980, la academia se percató de que las personas y las instituciones utilizan la traducción para distintos fines, que incluyen establecer, mantener y contrariar las estructuras de poder (Asimakoulas, 2011). Además, se dieron cuenta de que, en lugar de adherirse sin mayor reparo a los manuales de traducción, traductoras y traductores tomaron decisiones lingüísticas de manera creativa y con base en factores contextuales tanto implícitos como explícitos (Davies, 2011; Wolf, 2007; Yameng, 2007). Por tanto, se llegó a la conclusión de que la traducción es una actividad social que se ve afectada por las relaciones de poder que imperan en el contexto donde se lleva a cabo (Wolf, 2007; Yameng, 2007). Como se verá a continuación, este estudio confirma tales observaciones y conclusiones.

Como señalan Giovine (2010) y Vázquez (2009), en vista de que la Ley General de Derechos Lingüísticos de los Pueblos Indígenas no precisa con qué recursos ni quiénes se encargarán de realizar la traducción a lenguas indígenas de los instrumentos jurídicos con base en los cuales funciona el estado mexicano, no es de extrañar que, entre los obstáculos contextuales que surgieron en la realización de la traducción, se encuentren la falta de recursos materiales y humanos: la dependencia que gestionó la traducción no contaba con el presupuesto suficiente, por lo que tuvo que trabajar en conjunto con otra para que el proyecto pudiera llevarse a cabo; la dependencia gestora se compone, en su mayoría, de personas no hablantes de lenguas indígenas, por lo que la revisión estuvo a cargo de estudiantes de Antropología que realizan su servicio social en ella; el glosario existente no resultó de ayuda para el traductor y tuvo que elaborar uno propio; y el traductor no cuenta con herramientas que optimicen o faciliten la labor traductora como ocurre con las lenguas mayoritarias, por lo que hizo la traducción a mano y después la transcribió por computadora (Cano, 2017; Segundo, 2017). Estas limitantes dan cuenta del lugar que ocupan las lenguas indígenas y su traducción en la lista de prioridades del gobierno y de la sociedad mexicanos.

Por otra parte, en vista de que el Derechoy su discurso derivan de circunstancias políticas y sociales específicas, entre los obstáculos lingüísticos y culturales que el traductor enfrentó se encuentra la falta de equivalencias de términos como derecho, ministerio público, sistema jurídico, constitución y delito (Segundo, 2017). Esta falta de equivalencias demuestra que la redacción de la ley objeto de la traducción no considera la forma en que operan los sistemas jurídicos indígenas ni las circunstancias particulares de las mujeres indígenas, sino que se centra en la realidad de las mujeres no indígenas y en el funcionamiento exclusivo del aparato jurídico no indígena. Cabe decir, además, que el documento en español se dirige a las personas de la función pública, mientras que la traducción se dirige a mujeres indígenas, quienes, en la mayoría de los casos, no tienen conocimiento de la terminología jurídica (CIDH, 2017).

Para superar el obstáculo que supone la falta de equivalencias, el traductor dijo haber adaptado los términos jurídicos en español a la comprensión del mundo del pueblo mazahua. ${ }^{11}$ Además, dijo haber combinado ambas variantes del mazahua en la traducción porque, desde un posicionamiento comunitario, para él era fundamental que mujeres y hombres hablantes de ambas variantes sintieran que se les estaba incluyendo (Segundo, 2017). Esto resulta interesante, puesto que, para la dependencia gestora, el público meta son las mujeres mazahuas; ${ }^{12}$ sin embargo, para el traductor, lo era todo el pueblo. Este descubrimiento concuerda con el hecho de que, para las mujeres indígenas, la lucha es comunitaria y no individual, como tiende a promoverlo el feminismo hegemónico (Espinosa et al., 2014; Cerna Villagra, 2018).

Aunado a lo anterior, cabe decir que, para garantizar la comprensión del contenido de la ley, las dependencias participantes decidieron incluir el texto original en español

11 En consideración de que esta investigación constituyó un acercamiento social más que lingüístico, no se incluyen ejemplos de las técnicas traductoras que se emplearon.

12 El propósito de la traducción era informar a las mujeres indígenas acerca del contenido de la Ley de Acceso de las Mujeres a una Vida Libre de Violencia del Estado de México y, de esa forma, garantizar que sean capaces de identificar la violencia y de accionar los mecanismos que las protegen de ella. 
junto con la traducción (el texto aparece a dos columnas, español/masagua). El motivo — dijeron— es que la mayoría de las mujeres indígenas es bilingüe (español $<>$ lengua indígena), pero no sabe leer ni escribir en su lengua (Cano, 2017; Monroy, 2017). Además, mencionaron que llevan a cabo pláticas informativas con respecto al contenido de la traducción. En ese sentido, en vista de que la dependencia se compone principalmente de personas no hablantes de una lengua indígena, puede suponerse que tales pláticas se desarrollan en español. Así, se demuestra que la lengua de acceso sigue siendo la hegemónica.

Además, el traductor comentó que quienes saben leer y escribir el mazahua y el español son los hombres de la comunidad, pues son quienes tienen mayor contacto con el mundo no indígena. Tanto los niveles de analfabetismo (en español y en mazahua) como el limitado tiraje de traducciones limitarán el alcance de la traducción y su impacto en la vida de las comunidades. Además, en repetidas ocasiones, el traductor hizo hincapié en el hecho de que la ley es totalmente ajena a la realidad de su pueblo, pues se trata más bien de un instrumento con el cual se pretende asimilar a los pueblos indígenas a las prácticas sociojurídicas occidentalizadas (Segundo, 2017). Al respecto, mencionó lo siguiente:

En nuestra visión indígena entre lo masculino y femenino no existe exclusión, todo depende desde la mirada que se tenga de la dinámica social y cultural en que le mire. Falta un mayor entendimiento para ello, todo se quiere medir con el mismo rasero de manera homogénea. De tal forma que todos son incluyentes en la toma de decisiones que atañe a la familia y a la comunidad. Debe de verse el papel que juega hombre y mujer en la comunidad indígena. De tal forma que el mensaje de origen al texto meta no se nota ninguna discriminación ni exclusión (Segundo, 2017).

Si bien puede verse que el traductor defiende el sentido comunitario que caracteriza a los pueblos indígenas, resulta un tanto preocupante que minimice, de cierta manera, la violencia que reportan Olivares Ferreto et al. (2014) y Oehmichen (2005). En ese sentido, se espera que el análisis lingüístico - que se llevará a cabo como parte de la segunda etapa de esta investigación - revele si esta postura ideológica del traductor puede detectarse en las decisiones que haya tomado al realizar la traducción.

\section{Conclusiones}

Desde una perspectiva de los estudios descriptivos de traducción, los datos recabados hasta el momento sugieren que, en vista del contexto en el que se inserta, la traducción al mazahua de la Ley de Acceso de las Mujeres a una Vida Libre de Violencia del Estado de México juega un papel ambivalente. Por un lado, asienta - a perpetuidad — la voz de un pueblo marginado y subyugado, lo cual puede entenderse como una práctica de resistencia, oposición y diversificación (Cronin, 1998; Davies, 2011) que pudiera aunar a la revitalización de la lengua (Segundo, 2017; Monroy, 2017); por otro lado, la traducción constituye el instrumento por medio del cual el aparato estatal occidentalizado puede seguir interviniendo en la vida comunal de pueblos y personas indígenas so pretexto de la protección de la vida de las mujeres, es decir, puede entenderse como una herramienta de control, opresión y colonización (Cronin, 1998), en especial porque no considera, como lo señala la CIDH (2017), ni la visión ni la realidad ni el posicionamiento político de las mujeres indígenas y de sus pueblos (por ejemplo, no menciona nada sobre la protección del territorio).

Por lo anterior, puede concluirse que, mientras el gobierno mexicano no preste la debida atención a las prácticas comunicativas - sobre todo en contextos jurídicos-, y mientras se siga mostrando reacio a reconfigurar su relación, concepción y trato hacia pueblos, comunidades y personas indígenas, de manera que, como sujetos colectivos, dichos pueblos y comunidades puedan ejercer su derecho a la libre autodeterminación y puedan también relacionarse con el Estado como sugiere Villoro (2017), las prácticas que lleve a cabo el Estado en favor de una pretendida inclusión - como la traducción que nos ocupa- serán prácticas 'neoindigenistas ${ }^{\prime 13}$ cuyo impacto en la vida de las mujeres y en el mantenimiento de sus lenguas será nulo o limitado.

13 Para María Teresa Sierra (2005), las prácticas neoindigenistas son aquellas que aparentan incluir y respetar a las personas indígenas y a los pueblos a los que pertenecen, pero que, en realidad, son superficiales e, incluso, inútiles. 


\section{Referencias}

Adonon Viveros, A. (julio-diciembre, 2009). Estado, derecho y multiculturalismo. Un enfoque de antropología jurídica en México. Nueva Antropología, 22(71), 51-72.

Aguilar Gil, Y. E. (2018). Un nosotrxs sin estado. Tuxtla Gutiérrez, México: OnA Ediciones.

Aguilar Gil, Y. E. (septiembre, 2017). Ëëts, Atom. Algunos apuntes sobre la identidad indígena. Revista de la Universidad de México, 17-23.

Alberti Manzanares, P. (2004). ¿Qué es la violencia doméstica para las mujeres indígenas en el medio rural?. En T. Fernández de Juan (Coord.), Violencia contra la mujer en México (pp. 19-50). México, D. F., México: Comisión Nacional de los Derechos Humanos.

Aragón Andrade, O. (enero-abril, 2007). Los sistemas jurídicos indígenas frente al derecho estatal en México: Una defensa del pluralismo jurídico. Boletín Mexicano de Derecho Comparado, 40(118), 9-26.

Asimakoulas, D. (2011). Systems and the boundaries of agency: Translation as a site of opposition. In D. Asimakoulas \& M. Rogers (Eds.), Translation and opposition (pp. 1-36), Bristol, England: Multilingual Matters.

Bassnett, S. (2014). Translation studies (4a ed.). Suffolk, England: Routledge.

Cerna Villagra, S. (2018). Desafíos de la participación y representación política de las mujeres indígenas en México. En C. Wright (Coord.), Participación política indígena en México. Experiencias de gestión comunitaria, participación institucional y consulta previa (pp. 195-218). Ciudad de México, México: Itaca.

Chenaut, V. (diciembre, 2007). Género y justicia en la antropología jurídica en México. Papeles de Trabajo, (15), 47-72.

Comisión Interamericana de Derechos Humanos. (2017). Las mujeres indígenas y sus derechos en las Américas. Washington, D.C., Estados Unidos de América: OEA.

Conley, J. M., \& O'Barr, W. M. (2005). Just words: Law, language and power (2a ed.). Chicago, United States of America: University of Chicago Press.

Correas, O. (2012). Derecho indígena mexicano I ( $2^{\text {a }}$ ed.). Ciudad de México, México: Coyoacán.

Cronin, M. (1998). The cracked looking glass of servants: Translation and minority in a global age. In L. Venuti (Ed.), Translation and minority (pp. 145-162). Manchester, England: St. Jerome Publishing.
Davies, E. E. (2011). The translator's opposition: Just one more act of reporting. In D. Asimakoulas \& M. Rogers (Eds.), Translation and opposition (pp. 111-128). Bristol, England: Multilingual Matters.

De la Peña, G. (marzo, 2006). A new Mexican nationalism? indigenous rights, constitutional reform and the conflicting meanings of multiculturalism. Nations and Nationalism, 12(2), 279-302.

Equis Justicia para las Mujeres, Red Nacional de Abogadas Indígenas, Intersecta, Red Nacional de Refugios y Centro Profesional Indígena de Asesoría, Defensa y Traducción. (2019). Acceso a la justicia para las mujeres indígenas: Informe sombra para el comité de la ONU para la eliminación de la discriminación racial. México, D. F., México: EQUIS.

Espinosa Miñoso, Y., Gómez Correal, D. y Ochoa Muñoz, K. (2014). Tejiendo de otro modo: Feminismo, epistemología y apuestas descoloniales en Abya Yala. Popayán, Colombia: Universidad del Cauca.

Fernández Chagoya, M. (2016). Miradas multidisciplinarias en torno a la masculinidad: Desafíos para la impartición de justicia. Ciudad de México, México: Suprema Corte de Justicia de la Nación y Fontamara.

Gall, O. (abril-junio, 2004). Identidad, exclusión y racismo: Reflexiones teóricas y sobre México. Revista Mexicana de Sociología, 66(2), 221-259.

Gándara Carballido, M. (2019). Los derechos humanos en el siglo XXI: Una mirada desde el pensamiento crítico. Buenos Aires, Argentina: CLACSO.

Gémar, J. (2005). La asimetría cultural y el traductor jurídico. El lenguaje del Derecho, la cultural y la traducción. E. En Monzó Nebot y A. Borja Albi (Eds.), La traducción y la interpretación en las relaciones jurídicas internacionales (pp. 33-64). Castellón, España: Publicaciones de la Universitat Jaume I.

Genztler, E. (2002). Translation, poststructuralism, and power. In E. Genztler, \& M. Tymoczko (Eds.), Translation and power (pp. 195-218). Boston, United States of America: University of Massachusetts Press.

Gibbons, J. (2003). Forensic linguistics: An introduction to language in the justice system. Malden, United States of America: Blackwell Publishing.

Giovine Yáñez, M. A. (mayo, 2008). La diversidad etnolingüística en México en el marco de la Ley General de Derechos Lingüísticos de los Pueblos Indígenas. Potencia presentada en el IV congreso el español, lengua de traducción, Madrid, ES. 
González Galván, J. A. (2010). El estado, los indígenas y el derecho. México D. F., México: Universidad Nacional Autónoma de México.

Hernández, R. A. y Ortiz, H. (mayo, 2003). Diferentes pero iguales: Los pueblos indígenas en México y el acceso a la justicia. Conferencia presentada en Reforming the Administration of Justice in Mexico, California, US.

Instituto Nacional de Lenguas Indígenas. (2010). Catálogo de lenguas indígenas nacionales: Variantes lingüísticas de México con sus autodenominaciones y referencias geoestadísticas. México, D. F., México: INALI.

López Bárcenas, F. (2006). Autonomía y derechos indígenas en México. Bilbao, España: Universidad de Deusto.

López Bárcenas, F. (2010). Legislación y derechos indígenas en México (3a ed.). Ciudad de México, México: CEDRSSA y H. Cámara de Diputados.

Martí Sánchez, M. (2004). La compleja identidad del léxico jurídico. ELUA, (18), 169-189.

Meneses Méndez, D. (diciembre, 2004). ¿Palabras 0 conceptos?: La traducción en las lenguas mayas de México. En Consejo de Traductores e Intérpretes de Canadá y Asociación Cubana de Traductores e Intérpretes (Organizadores), V simposio de traducción, interpretación y terminología Cuba-Canadá. Simposio llevado a cabo en el Capitolio de La Habana, La Habana, CU.

Mooney, A. (2014). Language and law. London, England: Palgrave Macmillan.

Nord, C. (1988). Text analysis in translation: Theory, methodology and didactic application of a model for translation-oriented text analysis. Amsterdam, Países Bajos: Rodopi.

Ochoa Muñoz, K. (2019). Miradas en torno al problema colonial: Pensamiento anticolonial y feminismos descoloniales en los sures globales. Ciudad de México, México: Akal.

Oehmichen, C. (2005). Identidad, género y relaciones interétnicas. Mazahuas en la Ciudad de México. Ciudad de México, México: Universidad Nacional Autónoma de México.

Olivares Ferreto, E., Zermeño Núñez, F. y Domínguez Pérez, M. (2014). Violencia contra las mujeres indígenas universitarias a lo largo de su trayectoria en el ámbito educativo. Estudio de caso: Estudiantes de la Universidad Intercultural del Estado de México. Ciudad de México, México: Estudios y Estrategias para el Desarrollo y la Equidad.
Potapouchkine, N. y Haenisch, M. (2003). La terminología jurídica: Dificultades y estrategias de traducción. Revista de ciencias jurídicas, (08-09), 203-224.

Pym, A. (2006). Introduction: On the social and the cultural in translation studies. En A. Pym, M. Shlesinger \& Z. Jettmarová (Eds.), Sociocultural aspects of translating and interpreting (pp. 1-26). Amsterdam, Netherlands: John Benjamins Publishing Company.

Saldanha, G., \& O'Brien, S. (2013). Research methodologies in translation studies. Abingdon, England: Routledge.

Segato, R. L. (mayo-agosto, 2014). El sexo y la norma: Frente estatal, patriarcado, desposesión, colonialidad. Estudos Feministas, 22(2), 593-616.

Sierra, M. T. (2004). Haciendo justicia: Interlegalidad, derecho y género en regiones indigenas. Ciudad de México, México: Centro de Investigación y Estudios Superiores en Antropología Social.

Sierra, M. T. (agosto, 2005). Derecho indígena y acceso a la justicia en México: Perspectivas desde la interlegalidad. Revista IIDH, 41, 287-314.

Sierra, M. T. (septiembre-diciembre, 2009). Las mujeres indígenas ante la justicia comunitaria. Perspectivas desde la interculturalidad y los derechos. Desacatos, (31), 73-88.

Tiersma, P. M. (1999). Legal language. Chicago, United States of America: University of Chicago Press.

Toury, G. (1991). What are descriptive studies into translation likely to yield apart from isolated descriptions?. En K. van Leuven-Zwart \& T. Naaijkens (Eds.), Translation studies: The state of the art (pp. 179-192). Amsterdam, Netherlands: Rodopi.

Vázquez Carranza, A. (2009). Linguistic rights in Mexico. Revista Electrónica de Lingüística Aplicada, (8), 199-210.

Villoro, L. (2017). Ensayos sobre indigenismo: Del indigenismo a la autonomía de los pueblos indígenas. Madrid, España: Biblioteca Nueva.

Wolf, M. (2007). The emergence of sociology of translation. En M. Wolf \& A. Fukari (Eds.), Constructing a sociology of translation (pp. 1-38). Amsterdam, Netherlands: John Benjamins Publishing Company.

Yameng, L. (2007). Towards representational justice in translation practice. In J. Munday (Ed.), Translation as intervention (pp. 54-70). London, England: Continuum International Publishing Group. 